\title{
Outbreak of chronic renal failure: will this be a delayed heritage of COVID-19?
}

\author{
Niloofar Khoshdel-Rad ${ }^{1,2} \cdot$ Ensieh Zahmatkesh ${ }^{1,2} \cdot$ Anastasia Shpichka $^{3,4} \cdot$ Peter Timashev $^{3,4,5} \cdot$ Massoud Vosough $^{1,2}$
}

Published online: 2 September 2020

(c) Italian Society of Nephrology 2020

Keywords Acute kidney injury $\cdot$ Chronic renal failure · COVID-19 $\cdot$ Renal damage $\cdot$ SARS-CoV-2 $\cdot$ Coronavirus

$\begin{array}{ll}\text { Abbreviations } & \\ \text { ACE-2 } & \text { Angiotensin-converting enzyme 2 } \\ \text { AKI } & \text { Acute kidney injury } \\ \text { CRF } & \text { Chronic renal failure } \\ \text { ESRD } & \text { End-stage renal disease } \\ \text { RRT } & \text { Renal replacement therapy }\end{array}$

SARS-CoV-2 Severe acute respiratory syndrome coronavirus 2

Outbreaks of newly emerging infectious diseases have always threatened human health and global stability. The novel severe acute respiratory syndrome coronavirus 2 (SARS-CoV-2) is currently causing a global pandemic of a disease now named COVID-19. While the common symptoms are influenza-like, SARS-CoV-2 infection may lead to multiorgan dysfunction and death; fragile patients have, as in other viral diseases, a higher risk of adverse events. To date, there is no agreed treatment for COVID-19 and a wide range of treatments has been proposed, including antiviral

Peter Timashev

timashev.peter@gmail.com

$\triangle$ Massoud Vosough

masvos@royaninstitute.org

1 Department of Regenerative Medicine, Cell Science Research Center, Royan Institute for Stem Cell Biology and Technology, ACECR, Tehran, Iran

2 Department of Stem Cells and Developmental Biology, Cell Science Research Center, Royan Institute for Stem Cell Biology and Technology, ACECR, Tehran, Iran

3 Institute for Regenerative Medicine, Sechenov University, Moscow, Russia

4 Chemistry Department, Lomonosov Moscow State University, Moscow, Russia

5 Department of Polymers and Composites, NN Semenov Institute of Chemical Physics, Moscow, Russia drugs, immunotherapy, convalescent serum and monoclonal antibodies, cell-based therapies, and Chinese medicine [1].

The metallopeptidase angiotensin-converting enzyme 2 (ACE-2) is the functional receptor for SARS-CoV-2. It is present in various human organs such as heart, blood vessels, kidney, and gastrointestinal tract [2]. The kidneys are among the most common target organs of SARS-CoV-2. Several studies showed that renal deterioration is associated with 5.3 times in-hospital mortality risk in COVID-19 patients [3]. Computerized tomography (CT) scan of the kidneys showed that COVID-19 patients had renal interstitial inflammation and edema [3]. A broad range of clinical manifestations has been reported, including proteinuria (44-63\%), hematuria (26.9-44\%), urea (14.1-27\%) and serum creatinine increase (10-19\%). Moreover, up to $30 \%$ of hospitalized SARS-CoV2-infected patients developed acute kidney injury (AKI). As expected, pre-existing chronic kidney disease (CKD) was associated with a six-fold increased risk of AKI and severe COVID-19 infection [4]. Kidney involvement may in turn increase the risk of pulmonary edema, cardiopulmonary syndrome, circulatory insufficiency, and thromboembolic and hemorrhagic risks.

The pathogenesis of kidney involvement in COVID-19 patients is not fully understood but available data suggests that renal dysfunction may be multi-factorial: direct virus cytotoxicity, cytokine storm syndrome, thrombotic microangiopathy, and systemic hemodynamic changes seem to be the most important contributing factors (Fig. 1).

The SARS-CoV-2 has broad organotropism. Viral copies were found in different tissues including kidneys, liver, heart, brain, and blood. The SARS-CoV-2 virus enters into the cells through the viral structural spike (S) protein that binds to the ACE-2 receptors, which are broadly expressed in the podocytes, and mainly in proximal tubular cells. Following receptor binding onto renal cells, the fusion of the viral envelope with cellular membranes is mediated by a specific serine protease named TMPRSS2 (transmembrane 


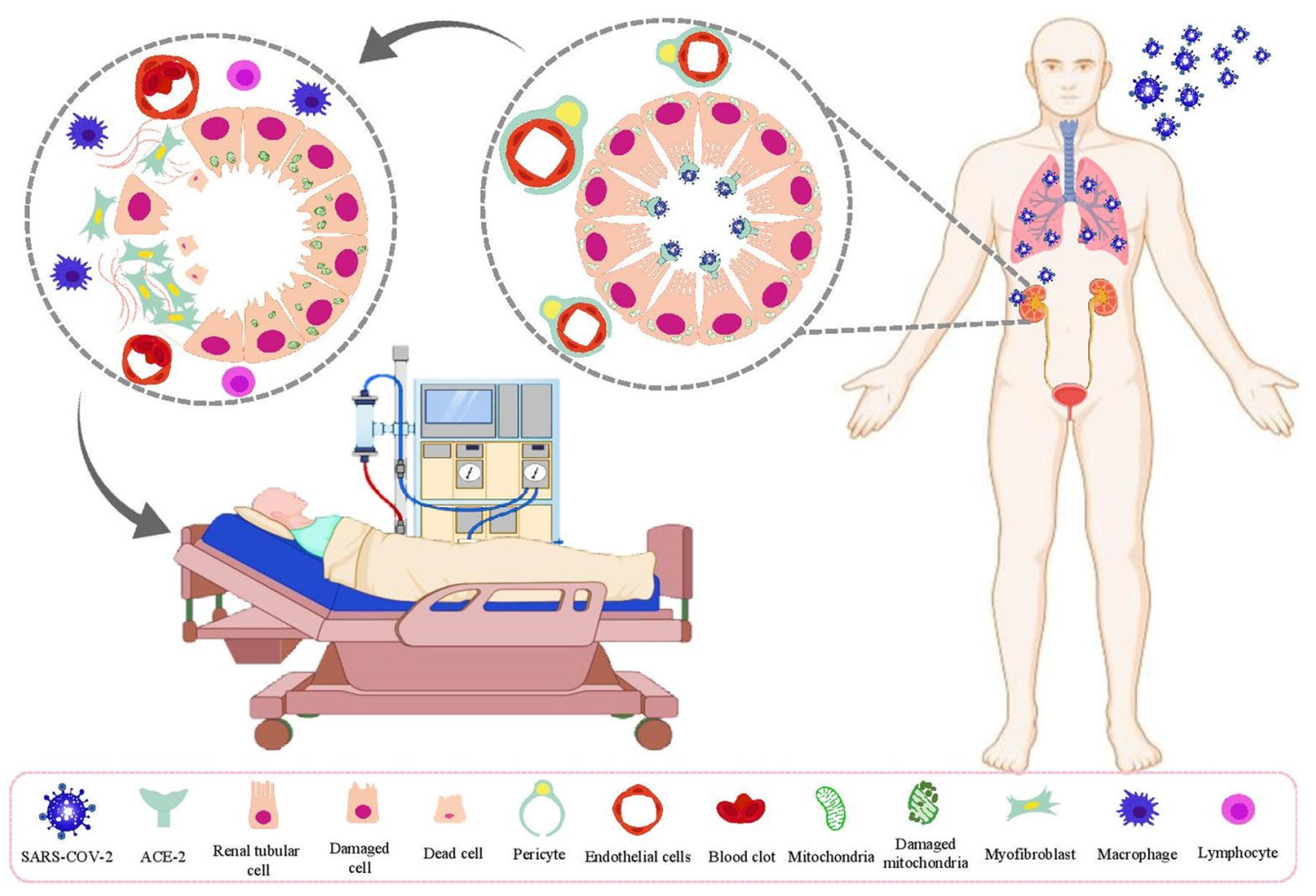

Fig. 1 Kidney injuries in COVID-19 patients. The SARS-CoV-2 virus enters into the renal cells through binding to the ACE-2 receptors. SARS-CoV-2 infection contributes to acute injuries including loss of brush border, lumen dilation, disruption of mitochondria, and necrosis in tubular proximal cells. Moreover, COVID-19 is related to the detachment of perivascular pericytes, sub-endothelial space

serine proteinase 2) co-expressed with ACE-2 in the renal cells [5]. These events are associated with foot process defacement, detachment of podocytes from the glomerular basement membrane, and collapsing glomerulopathy. In addition, loss of brush border, vacuolar degeneration, lumen dilation, disruption of mitochondria and necrosis in tubular proximal cells have been reported [6]. Furthermore, scattered macrophage and lymphocytic infiltrates in some areas such as the sub-capsular space of kidney tissues, and edematous expansion of the interstitial space in both distal tubules and collecting duct segments were reported in the context of cytokine storm [6]. Considering that the kidneys receive approximately $25 \%$ of the cardiac output, hypoxia may further contribute to the kidney damage. Evidence of endothelial injury such as pericyte detachment, sub-endothelial space expansion, endothelial proliferation without deposits, and foam cell accumulation, (endothelial damages and thrombosis) were also reported [6].

Observational studies, performed in contexts other than COVID-19, examining patients with AKI from different causes, found that a relevant number of survivors developed chronic renal failure (CRF) during medium-term follow-up expansion, erythrocyte aggregation, and blood clot formation in the intra-renal vasculature. Incomplete recovery from AKI, continuous interstitial inflammation, the loss of the regenerative potential of renal vascular cells, and hypertension are often related to the increased risk of CRF progression and dialysis during the long-term periods after hospitalization periods (1-5 years after hospitalization) [7]. The risk of $\mathrm{CKD}$ is associated with the severity of AKI and with the diagnosis of acute tubular necrosis [8]. Potential pathophysiological mechanisms include incomplete recovery, continuous interstitial inflammation, loss of regenerative potential of renal vascular cells, and hypertension.

Emerging data indicate infection by SARS-CoV-2 leads to acute and chronic kidney tissue damage via acute tubular necrosis, interstitial inflammation, renal vasculature injuries, and collapsing glomerulopathy [6]. Furthermore, the severity of COVID-19 is associated with risk of AKI and need for dialysis [9].

It is therefore conceivable that patients who survive COVID-19-related AKI are at increased risk of developing progressive $\mathrm{CKD}$ after recovery from the viral infection. The high cost of renal replacement therapy and the lack of uniform availability of dialysis facilities, make this possibility very scary due to the widespread difficulty in increasing the health-care budgets for the treatment of kidney diseases.

With this editorial we would like to stress that it is of fundamental importance that clinicians pay attention to the evaluation of renal function among COVID-19 survivors, 
even among those with apparent recovery, to reduce chronic kidney impairment and avoid progression to end-stage renal disease (ESRD). Follow-up with a nephrologist and the identification of pathological conditions associated with the outcomes is fundamental to understanding the natural history of the disease and to improve the outcome in these patients population, and may also represent a cost effective strategy for controlling the global expenditure for advanced CKD and renal replacement therapy (RRT).

Acknowledgements This work was partly supported by the Russian academic excellence project 5-100.

\section{Compliance with ethical standards}

Conflict of interest The authors declare no conflicts of interest.

Ethical approval This article doesn't contain any studies with human participants or animals performed by any of the authors.

Informed consent No required.

\section{References}

1. Hossein-khannazer N, Shokoohian B, Shpichka A, Aghdaei HA, Timashev P, Vosough M (2020) Novel therapeutic approaches for the treatment of COVID-19. J Mol Med 98:789-803

2. Monteil V, Kwon H, Prado P, Hagelkrüys A, Wimmer RA, Stahl $M$ et al (2020) Inhibition of SARS-CoV-2 infections in engineered human tissues using clinical-grade soluble human ACE2. Cell 181(4):905-913.e7

3. Li Z, Wu M, Yao J, Guo J, Liao X, Song S et al (2020) Caution on kidney dysfunctions of COVID-19 patients. MedRxiv. https:// doi.org/10.1101/2020.02.08.20021212

4. Henry BM, Lippi G (2020) Chronic kidney disease is associated with severe coronavirus disease 2019 (COVID-19) infection. Int Urol Nephrol 52(6):1193-1194. https://doi.org/10.1007/s1125 5-020-02451-9

5. Su H, Yang M, Wan C, Yi LX, Tang F, Zhu HY et al (2020) Renal histopathological analysis of 26 postmortem findings of patients with COVID-19 in China. Kidney Int. https://doi.org/10.1016/j. kint.2020.04.003

6. Pan XW, Xu D, Zhang H, Zhou W, Wang LH, Cui XG (2020) Identification of a potential mechanism of acute kidney injury during the COVID-19 outbreak: a study based on single-cell transcriptome analysis. Intensive Care Med 46:1114-1116

7. Bassegoda O, Huelin P, Ariza X, Solé C, Juanola A, GratacósGinès J et al (2020) Development of chronic kidney disease after acute kidney injury in patients with cirrhosis is common and impairs clinical outcomes. J Hepatol 72(6):1132-1139. https:// doi.org/10.1016/j.jhep.2019.12.020

8. Schiffl H, Fischer R (2012) Clinical cause of presumed acute tubular necrosis requiring renal replacement therapy and outcome of critically ill patients: post hoc analysis of a prospective 7-year cohort study. Int Urol Nephrol 44(6):1779-1789

9. Liu Y-F (2020) The chronic kidney disease and acute kidney injury involvement in COVID-19 pandemic a systematic review and meta-analysis. MedRxiv. https://doi. org/10.1101/2020.04.28.20083113

Publisher's Note Springer Nature remains neutral with regard to jurisdictional claims in published maps and institutional affiliations. 